\title{
Liposuction in Cancer-Related Lower Extremity Lymphedema: An Investigative Study For Clinical Applications
}

Jianfeng Xin

Capital Medical University Affiliated Beijing Shijitan Hospital

\section{Yuguang Sun}

Capital Medical University Affiliated Beijing Shijitan Hospital

\section{Song Xia}

Capital Medical University Affiliated Beijing Shijitan Hospital

Kun Chang

Capital Medical University Affiliated Beijing Shijitan Hospital

Chao Dong

Sanfine International Hospital

\section{Zhong Liu}

Capital Medical University Affiliated Beijing Shijitan Hospital

\section{Jian Dong}

Capital Medical University Affiliated Beijing Shijitan Hospital

Wenbin Shen ( $\nabla$ shenwb@bjsjth.cn )

Beijing Shijitan Hospital,Capital Medical University

\section{Research}

Keywords: lymphedema, lower extremity, liposuction

Posted Date: November 23rd, 2021

DOl: https://doi.org/10.21203/rs.3.rs-559817/v2

License: (9) (1) This work is licensed under a Creative Commons Attribution 4.0 International License. Read Full License

Version of Record: A version of this preprint was published at World Journal of Surgical Oncology on January 5th, 2022. See the published version at https://doi.org/10.1186/s12957-021-02472-3. 


\section{Abstract}

Background: Lymphedema is a progressive, noncurable condition consisting of increases in subcutaneous fat and interstitial fluid in the limbs and fibrosis during later stages. The disease most commonly affects the limbs because of injury to or removal of lymph nodes. The aim of this study was to investigate therapeutic outcomes of liposuction for cancer-related lower extremity lymphedema.

Methods: 62 patients with cancer-related lymphedema in unilateral lower extremity were recruited in this study, and all the patients underwent liposuction. The volume of hemorrhage and lipid, the operation time, and the volume changes of the affected extremity were compared by applying $t$-test, and the subjective feelings of patients were also assessed by applying Chi-square.

Results: Total lipid volume is $(2539 \pm 1253.5) \mathrm{ml}$, and the hemorrhage volume is $(828 \pm 311.8) \mathrm{ml}$ during liposuction. For the comparison of objective indexes, (1) Percent volume differences(PVDs) before

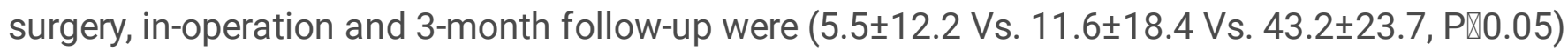
respectively. (2) Higher lipid volume and liposuction rate for female patients, with a lower volume of hemorrhage. (3) Higher volume of hemorrhage in patients with a history of recurrent erysipelas. (4) Higher lipid volume and liposuction rate(LR), with lower hemorrhage for stage II than stage III patients.

Conclusions: Liposuction is an effective therapy for cancer-related lower extremity lymphedema. Gender, stage and recurrent erysipelas history influence the course and effect of liposuction.

\section{Introduction}

Pelvic tumor surgery is traditionally characterized by several major complications, of which secondary lower extremity lymphedema is one. The patients with cancer-related lower extremity lymphedema are associated with its treatment modalities, such as cancer surgery and radiation therapy, which can injury to or removal of lymph nodes[1]. Lymphedema is a disabling condition whereby damage to lymphatic channels causes the accumulation of protein-rich lymphatic fluid with the subcutaneous tissues which can cause abnormal proliferation of subcutaneous adipose tissue[2].Liposuction refers to a surgical technique that uses various types of equipment to aspirate excess subcutaneous fat through small incisions made on the skin to improve body esthetics, and pressure garments are to be worn for 3 to 6 months after liposuction[3]. Since lllouz first reported the negative pressure suction method for fat aspiration in 1983, liposuction has been widely applied in various therapeutic fields[4]. The most common area for fat removal is the abdomen, flanks, trochanteric region, lumbar region, gluteal region, thighs, and calves[5].The therapeutic effect of liposuction for lymphedema is still controversial. Brorson has reported that liposuction combined with post-operative controlled compression therapy (CCT) is effective for the treatment of limb lymphedema[6]. Chen and Chollet have found that liposuction without concurrent skin excision, the skin often does not retract sufficiently and can lead to wound healing complications[7, 8]. However, the research results of Qi showed that the limb volume reduction was noted in all cases immediately after the operation, but this state of volume reduction remained 3 to 12 months 
with a mean of 6.01 months by applying low-stretch bandages or compression stockings. Subsequently, it returned to the preoperative volume level[9]. Other researchers have found that liposuction can significantly reduce lymphedema limb volume, but excess extracellular fluid persists[10].In addition, the potential surgical risks and the effects in different lymphedema patient groups have not yet been investigated.

In order to investigate the therapeutic effects of liposuction, the volume of aspirated fat(VAF) and the volume of blood loss(VBL) during the operation in different patient groups with cancer-related lower extremity lymphedema was evaluated. And the volume difference(VD) before surgery, in-operation and 3 months after the operation was compared.

\section{Materials And Methods}

\section{Study population}

The patients with lymphedema admitted to Beijing Shijitan Hospital from January to June 2017 were reviewed, and the inclusion criteria were as follows:(1)Only unilateral lower extremity was attacked by secondary lymphedema only affected; (2) according to the lymphedema staging established by the International Society of Lymphology (ISL) in 2013[11], patients were classified as either stage II or stage III; (3) the initial operation was liposuction; (4) postoperative compression stockings wearing were mandatory; (5) patients were followed up for more than 3 months. Exclusion criteria: (1) patients with a history of surgical treatment for lymphedema;(2)lower extremity lymphedema treated by liposuction combined with other surgical methods simultaneously. This retrospective study was conducted after approval by the Institutional Review Board of Beijing Shijitan Hospital, and a waiver of consent was obtained.

\section{Surgical technique}

The operation was performed under general anesthesia with endotracheal intubation. The tumescent solution was infused into subcutaneous adipose tissue. Liposuction equipment (GZXZ resonance liposuction system, produced by YangguangZhongtian Medical Equipment Co., Ltd. in Shanxi) was used to suck subcutaneous adipose and interstitial fluid. At the end of the operation, a Jackson-Pratt (JP) drain was placed subcutaneously to drain. Then, the operated limb was wrapped in compression bandages. On postoperative day 3 , after dressing change and drain removal, a compression garment was used continuously. The patient was allowed to do rehabilitation exercises.

\section{Measurement}

$B M I\left(\right.$ Body Mass Index) $. \mathrm{BMI}=$ weight $(\mathrm{kg}) /$ height $^{2}\left(\mathrm{~m}^{2}\right)$.

VD and PVD Based on limb measurement and volume calculation method devised by Brorson $\mathrm{H}[12]$

VD =affected limb volume - healthy limb volume; 
PVD $=[($ affected limb volume - healthy limb volume $) /$ healthy limb volume $] \times 100 \%$

VBL Pre-operative hemoglobin, post-operative hemoglobin, and volume of intra-operative blood transfusion were recorded. Pre-operative blood volume was obtained based on the formula derived by Nadler et al[13]. Subsequently, the volume of blood loss was calculated according to the formula derived by Budny et al[14].

VBL $(\mathrm{ml})=$ pre-operative blood volumex [(pre-operative hemoglobin囚post-operative hemoglobin $) \div$ preoperative hemoglobin] $\varangle$ volume of intra囚operative blood transfusion

The Liposuction Rate(LR) LR= VAF/ duration of operation.

\section{Postoperative follow-up}

Limb shaping was completed 3 to 6 months postoperatively[15], so the follow-up was carried out 3 months after the operation. Patient follow-up consisted of measuring BMI, PVD. And patients filled out a questionnaire to evaluate their subjective feelings, which encompassed stiffness, tension, heaviness, tenderness, pain, numbness, fatigue, and weakness.

\section{Statistical Analysis}

Data were presented as mean \pm standard deviation(Mean $\pm S D$ ). Kolmogorov-Smirnov test was performed to verify that the parameters conformed to normal distribution. The means of the two samples were compared by applying a $t$-test of independent samples. PVDs of the samples were compared by applying a $t$-test of paired samples. A corrected $t$-test was employed when there was a lack of variance. Chi-square was utilized to test the rate. $P<0.05$ was considered statistically significant. The data were analyzed using SPSS version 20.0 (IBM, Armonk, New York, USA).

\section{Results}

\section{Patient characteristics}

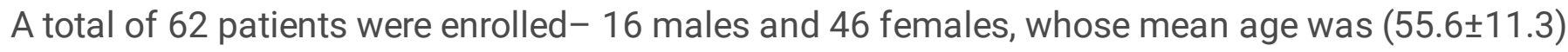
years(range 21-75). The mean duration of the disease was 55.8 months(range 12-288). Twenty-nine

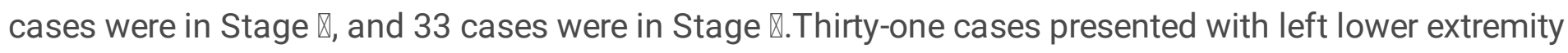
lymphedema, and 31 cases with right lower extremity lymphedema. Twenty patients had a history of recurrent erysipelas. The causes of the lymphedema included lymphedema secondary to cervical cancer in 29 cases, endometrial cancer in 11 cases, inguinal stromal tumor in 8 cases, ovarian cancer in 3 cases, testicular cancer in 3 cases, lymphoma in 3 cases, rectal cancer in 2 cases, penile cancer in 2 cases and bladder cancer in 1 case.

\section{Surgical outcomes}


In this study, 62 cases were operated successfully without skin necrosis, subcutaneous effusion, infection, or other complications(Figure1b, Figure2b). The operation time was (176 \pm 44.9$)$ min(range 110$300)$. The VAF was $(2539 \pm 1253.5) \mathrm{ml}($ range $400-5,500)$. The estimated blood loss (EBL) was $(828 \pm 311.8) \mathrm{ml}($ range224-1,585). Intra-operative homologous blood transfusion was performed in 3 patients. The LR was $14.8 \pm 7.7 \mathrm{ml} / \mathrm{min}$ (range 1.0-30.0). The appearance of the lymphedematous extremity significantly improved at postoperative 3 months. Preoperative BMI was $26.7 \pm 4.2$, and postoperative 3 months $\mathrm{BMI}$ was $25.9 \pm 4$.0. The difference was not statistically significant $(\mathrm{t}=1.038$, $P=0.301)$.

PVD at pre-operation, post-operation, and 3 months follow-up were $(43.2 \pm 23.7) \%,(5.5 \pm 12.2) \%$ and (11.6 \pm 18.4$) \%$,respectively.PVD at post-operation and 3-month follow-up significantly decreased compared with that at pre-operation $(P<0.05)$, but it significantly increased at 3-month follow-up compared with that at post-operation $(P<0.05)$.

VAF and LR were higher in females than those in males; whereas EBL, PVD at pre-operation, postoperation and 3 months follow-up were higher in males than those in females(Table 1). EBL was higher in the recurrent erysipelas group than that in the non-erysipelas group(Table 2).The VAF and LR were higher in Stage II than those in Stage III group. In contrast, EBL, PVD at pre-operation, post-operation, and 3 months follow-up were lower in the Stage II group (Table 3).

Table 1

Comparison of surgical results between male and female patients with secondary lymphedema of lower extremities (Mean \pm SD)

\begin{tabular}{|lllll|}
\hline & Male & Female & t & Pvalue \\
\hline N & 16 & 46 & - & - \\
\hline VBL $(\mathrm{ml})$ & $986 \pm 346$ & $774 \pm 282$ & 2.434 & 0.018 \\
\hline VAF $(\mathrm{ml})$ & $1659 \pm 1223$ & $2845 \pm 1122$ & -3.559 & 0.001 \\
\hline LR $(\mathrm{ml} / \mathrm{min})$ & $8.8 \pm 5.9$ & $16.9 \pm 7.2$ & -4.013 & $<0.001$ \\
\hline PVD & & & \\
\hline pre-operation (\%) & $56.8 \pm 22.1$ & $38.4 \pm 22.6$ & 2.807 & 0.007 \\
\hline post-operation (\%) & $16.5 \pm 13.7$ & $1.7 \pm 9.1$ & 4.034 & 0.001 \\
\hline 3 month follow-up (\%) & $22.9 \pm 18.6$ & $7.7 \pm 16.9$ & 3.024 & 0.004 \\
\hline N numbers; VBL volume of blood loss; VAF volume of aspirated fat; LR liposuction rate; \\
PVD percent volume difference
\end{tabular}


Table 2

Comparison of surgical results between recurrent erysipelas and non-erysipelas group with secondary lymphedema of lower extremities(Mean \pm SD)

\begin{tabular}{|lllll|}
\hline & recurrent erysipelas & non-erysipelas & t & P value \\
\hline $\mathrm{N}$ & 20 & 42 & - & - \\
\hline $\mathrm{VBL}(\mathrm{ml})$ & $1000 \pm 315$ & $747 \pm 278$ & 3.201 & 0.002 \\
\hline $\mathrm{VAF}(\mathrm{ml})$ & $2982 \pm 1412$ & $2328 \pm 1127$ & 1.965 & 0.054 \\
\hline LR (ml/min) & $16.3 \pm 7.7$ & $14.1 \pm 7.7$ & 1.057 & 0.295 \\
\hline PVD & & & & \\
\hline pre-operation (\%) & $49.0 \pm 27.5$ & $40.4 \pm 21.5$ & 1.343 & 0.184 \\
\hline post-operation (\%) & $7.3 \pm 13.7$ & $4.6 \pm 11.5$ & 0.808 & 0.422 \\
\hline 3 month follow-up (\%) & $14.9 \pm 17.9$ & $10.1 \pm 18.7$ & 0.951 & 0.346 \\
\hline Abbreviations: see Table 1 & & & & \\
\hline
\end{tabular}

Table 3

Comparison of surgical results between stage II and stage III group with secondary lymphedema of lower extremities(Mean \pm SD)

\begin{tabular}{|lllll|}
\hline & stage II & stage III & t & P value \\
\hline $\mathrm{N}$ & 29 & 33 & - & - \\
\hline $\mathrm{VBL}(\mathrm{ml})$ & $689 \pm 249$ & $950 \pm 313$ & -3.598 & 0.001 \\
\hline VAF $(\mathrm{ml})$ & $2875 \pm 1247$ & $2243 \pm 1199$ & 2.031 & 0.047 \\
\hline LR (ml/min) & $18.0 \pm 8.4$ & $12.1 \pm 6.0$ & 3.189 & 0.002 \\
\hline PVD & & & & \\
\hline pre-operation (\%) & $28.1 \pm 18.4$ & $56.5 \pm 19.7$ & -5.821 & $<0.001$ \\
\hline post-operation (\%) & $-3.2 \pm 6.2$ & $13.3 \pm 10.9$ & -7.438 & $<0.001$ \\
\hline 3 month follow-up (\%) & $1.7 \pm 15.3$ & $20.4 \pm 16.6$ & -4.576 & $<0.001$ \\
\hline Abbreviations: see Table 1 & & & & \\
\hline
\end{tabular}

The feeling of heaviness and fatigue of the operated limb was alleviated at 3-month follow-up compared with that at pre-operation, whereas feelings of stiffness, tenderness and tightness worsened. There were no significant differences in pain, numbness and weakness between pre-operation and 3- month followup(Table 4). 
Table 4

Comparison of subjective sensation between pre-operation and 3 months follow-up in 62 patients with secondary lymphedema of the lower extremity [case (\%)]

\begin{tabular}{|lllll|}
\hline & pre-operation & 3 month follow-up & X2 & P value \\
\hline stiffness & $25(40.3)$ & $36(58.1)$ & 3.904 & 0.048 \\
\hline tightness & $19(30.6)$ & $35(56.4)$ & 8.398 & 0.004 \\
\hline heaviness & $49(80.6)$ & $34(54.8)$ & 9.448 & 0.002 \\
\hline tenderness & $5(7.9)$ & $13(21.0)$ & 4.305 & 0.038 \\
\hline pain & $6(9.7)$ & $13(21.0)$ & 3.046 & 0.081 \\
\hline numbness & $33(53.2)$ & $41(66.1)$ & 2.145 & 0.143 \\
\hline fatigue & $44(71.0)$ & $27(43.5)$ & 9.523 & 0.002 \\
\hline weakness & $7(11.3)$ & $11(17.7)$ & 1.040 & 0.308 \\
\hline
\end{tabular}

\section{Discussion}

In the world, secondary lymphedema most commonly occurs following treatments of cancer. A variety of conservative therapies have been reported, including complete decongestion therapy(CDT)[16], as well as microsurgical reconstruction including lymphatic venous anastomosis[17] or lymph node transplantation[18, 19].Failure of these treatments to provide a complete reduction in patients with longstanding pronounced lymphedema is due to the persistence of excess newly formed subcutaneous adipose tissue in response to slow or absent lymph flow, which is not removed in patients with chronic lymphedema. Liposuction has drawn increased attention in the surgical treatment of lymphedema because it can remove hypertrophied adipose tissue.

Here we have demonstrated that liposuction surgery improved both quality of life and volumetric measurements in patients with lymphedema. Overall, the PVD was significantly lower at post-operation and 3 months follow-up than that at pre-operation. The mean estimated blood loss (EBL) was $828 \mathrm{ml}$, which was much higher than the EBL of $292 \mathrm{ml}$ when liposuction was done in obese patients[20]. The reason may be that there is abnormal hyperplasia of subcutaneous adipose tissue in lymphedematous limbs and that the proliferating adipose layer contains less regular fascial septae, which poses more difficulty during liposuction of lymphedematous limbs, aggravates peripheral vascular injury and increases bleeding than conventional liposuction. Therefore, patients with anemia should be proactively corrected before liposuction. Some patients may need packed red blood cells (pRBC) prepared before surgery. In this study, 3 patients were given an intra-operative homologous blood transfusion.

Male had a significantly higher PVD than female at pre-operation, post-operation, and 3 months followup, but significantly lower VAF and higher EBL. This suggests that there may be differences between 
males and females in the occurrence and development of lymphedema. Perhaps the abnormality of the lymphedematous lower extremity in males is more profound than that in females. In addition, subcutaneous tissue hyperplasia and fibrosis may aggravate as the disease progresses. We also found that the LR in males was significantly lower than that in females during the operation. That is, it was more difficult to extract fat in males. Moreover, the effect of different hormone levels between males and females on the composition of subcutaneous tissue[21, 22], as well as poor compliance of males may account for the aforementioned differences, which need to be further investigated.

Lymphedema is one of the risk factors for the occurrence of erysipelas[23].Recurrent erysipelas can lead to subcutaneous tissue fibrosis, which in turn can aggravate lymphedema. In the study of Kosenkov, a correlation was found between the occurrence of erysipelas and the degree of lymphedema, and they aggravated each other[24]. In our study, the recurrent erysipelas group was noted to have higher EBL than the non-erysipelas group. This suggests that patients with recurrent erysipelas may have more fibrosis in the subcutaneous tissue, which could increase the difficulty and risk of the surgery.

The PVD of the Stage $\otimes$ group was significantly higher than that of the Stage $₫$ group at pre-operation, post-operation and 3-month follow-up, whereas the VAF and LR of the Stage III group was significantly lower than those of the Stage II group. In addition, the EBL of the Stage III group is greater than that of the Stage II group. These results suggest that the longer the disease period, the more difficult the liposuction may become, and the more blood loss may ensue. It can be deduced that the surgical outcome may also be worse. The reason may be that the degree of fibrosis of subcutaneous tissue gradually elevates along the course of the disease. Sun et al. also believe that skin hardness is positively correlated with the stage of lymphedema[25]. Consequently, the outcome of liposuction and volume reduction in advanced limb lymphedema may not be as good as those in early lymphedema.

Regarding subjective feelings of the patients, the feeling of heaviness and fatigue of the affected limbs significantly reduced at 3-month follow-up compared with that at pre-operation, but the feeling of stiffness, tenderness and tightness of the lymphedematous limb was more severe than that at preoperation. This may be related to the injury of subcutaneous nerves and inflammatory reactions after liposuction. It is worth noting that $58.1 \%$ of patients still felt stiff, $56.4 \%$ felt tight and $54.8 \%$ felt heavy 3 months after liposuction. The reason may be that the compression stocking fails to effectively improve lymphatic reflux despite the significantly reduced volume of the lymphedematous limb after liposuction.

The data also show that PVD increases significantly at 3-month follow-up after liposuction, which is consistent with the subjective sensory changes of the patients. Qi et al. compared the outcome of liposuction in 17 patients(7 upper limb lymphedema and 10 lower limb lymphedema). Substantial limb volume reduction was noted in all cases immediately after operation. This state of volume reduction remained 3 to 12 months with a mean of 6.01 months by applying low-stretch bandages or compression stockings. Subsequently, it returned to the preoperative volume level[9]. This implies that liposuction combined with more effective post-operative measures to ameliorate the reflux of lymph could contribute to a better surgical outcome of extremity lymphedema. 


\section{Conclusions}

Liposuction demonstrates certain positive effects on the treatment of the cancer-related lower extremity lymphedema. Nevertheless, compared with traditional liposuction in plastic surgery, it is more risky and difficult. Moreover, gender differences, history of erysipelas and stage of the disease should be taken into account during the treatment process, especially during the perioperative period.

\section{Abbreviations}

EBL: mean estimated blood loss; CCT: controlled compression therapy ;VBL:volume of blood loss;VAF: volume of aspirated fat; LR: liposuction rate; PVD: percent volume difference; BMI :body mass index

\section{Declarations}

\section{Ethics approval and consent to participate}

Any repository data used in this study are open access and do not require any permissions. Ethics approval and consent to participate are not applicable for them.

\section{Consent for publication}

Not applicable.

\section{Availability of data and materials}

Not applicable.

\section{Competing interests}

The authors declare that they have no competing interests.

\section{Funding}

This project was supported by Beijing Municipal Administration of Hospitals Incubating Program $\rrbracket$ code:PX2022028. The funder is the corresponding author of the study and is responsible for the overall design and planning of this study.

\section{Authors' contributions}

All authors made substantial contributions to the conception and design of the study. The authors read and approved the final manuscript.

\section{Acknowledegments}

Not applicable. 


\section{References}

1. Bernas $M$, Thiadens S, Smoot B,Armer JM, Stewart P, Granzow J. Lymphedema following cancer therapy: overview and options. Clinical \& Experimental Metastasis, 2018; 35(5-6):547-551.

2. Dayan JH, Ly CL, Kataru RP, MehraraBJ. Lymphedema: Pathogenesis and Novel Therapies. Annual review of medicine. 2018; 69(1): 263-276.

3. D.Dhami L. Liposuction. Indian J Plast Surg. 2008 Oct; 41(Suppl): S27-S40.

4. Illouz YG. Body contouring by lipolysis: a 5-year experience with over 3000 cases. Plast Reconstr Surg. 1983;72(5):591-597.

5. Wu S, Coombs DM, Gurunian R. Liposuction: Concepts, safety, and techniques in body-contouring surgery. Cleveland Clinic journal of medicine.2020;87(6):367-375.

6. Brorson H. From Lymph to Fat: Liposuction as a Treatment for Complete Reduction of Lymphedema. International Journal of Lower Extremity Wounds. 2012; 11(1):10-19.

7. Chen WF, Zeng WF, Hawkes PJ, Jeanette M, Bowen M. Lymphedema Liposuction with Immediate Limb Contouring. Plastic and Reconstructive Surgery Global Open. 2019; 7(11):e2513.

8. Chollet C,Malloizel-Delaunay J,Cabarrou B, Chantalat E, Leray H, Garmy-Susini B, Yannoutsos A, Chaput B, Vaysse C. Liposuction-assisted brachioplasty in breast cancer-related lymphedema: Impact on volume reduction and quality of life. J Plast Reconstr Aesthet Surg.2021;74(7):16331701.

9. Qi FZ, Yang YW, Gu JY, SHI YD. The Long Term Efficacy of the Pure Liposuction Treatment for Patiens with Limb Lymphoedema. Clinical Medical Journal of China.2008;15(1):89-91.

10. Boyages J, Kastanias K, Koelmeyer LA, Winch CJ, Mackie H. Liposuction for advanced lymphedema: a multidisciplinary approach for complete reduction of arm and leg swelling. Ann Surg Oncol.2015;22 Suppl 3:S1263-S1270.

11. International Society of Lymphology. The diagnosis and treatment of peripheral lymphedema: 2013 Consensus Document of the International Society of Lymphology. Lymphology.2013;46(1):1-11.

12. Brorson H, Hoijer P. Standardised measurements used to order compression garments can be used to calculate arm volumes to evaluate lymphoedema treatment. J Plast Surg Hand Surg. 2012;46(6):410-415.

13. Nadler SB, Hidalgo JH, Bloch T. Prediction of blood volume in normal human adults. Surgery. 1962;51(2):224-232.

14. Budny PG, Regan PJ, Roberts AH. The estimation of blood loss during burns surgery. Burns.1993;19(2):134-137.

15. Mendez BM, Coleman JE, Kenkel JM. Optimizing Patient Outcomes and Safety With Liposuction. Aesthetic Surgery Journal.2019;39(1):66-82.

16. Tambour M, Holt M, Speyer A, Christensen R, Gram B. Manual lymphatic drainage adds no further volume reduction to Complete Decongestive Therapy on breast cancer-related lymphoedema: a multicentre, randomised, single-blind trial. Br J Cancer. 2018;119(10):1215-1222. 
17. AlJindan FK, Lin CY, Cheng MH. Comparison of Outcomes between Side-to-End and End-to-End Lymphovenous Anastomoses for Early-Grade Extremity Lymphedema. Plast Reconstr Surg. 2019;144(2):486-496.

18. Gould DJ, Mehrara BJ, Neligan P, Cheng MH, Patel KM. Lymph node transplantation for the treatment of lymphedema. J Surg Oncol. 2018;118(5):736-742.

19. Koide S, Lin CY, Chen C, Cheng MH. Long-term outcome of lower extremity lymphedema treated with vascularized lymph node flap transfer with or without venous complications. J Surg Oncol. 2020;121(1):129-137.

20. Swanson E. Does laser assistance reduce blood loss after liposuction? Aesthet Surg J. 2015;35(2):38-39.

21. de la Casa AM, Suarez SC, Rebollo RJ, JiménezRejano. Cellulite's aetiology: a review. J Eur Acad Dermatol Venereol.2013;27(3):273-278.

22. Xiao Z, Guo B, Gong J, Tang Y, Shang J, Cheng Y, Xu H. Sex-and age-specific percentiles of body composition indices for Chinese adults using dual-energy $\mathrm{X}$-ray absorptiometry. Eur $\mathrm{J}$ Nutr.2017;56(7):2393-2406.

23. Inghammar M, Rasmussen M, Linder A. Recurrent erysipelas-risk factors and clinical presentation. BMC Infect Dis.2014;14:270.

24. Kosenkov AN, Narenkov VM, Abramov I. Erysipelas as the cause of lymphedema. Khirurgiia (Mosk).2005(11):51-53.

25. Sun D, Yu Z, Chen J, Wang L, Han L, Liu N. The value of using a SkinFibroMeter for diagnosis and assessment of secondary lymphedema and associated fibrosis of lower limb skin. Lymphat Res Biol.2017;15(1):70-76.

\section{Figures}




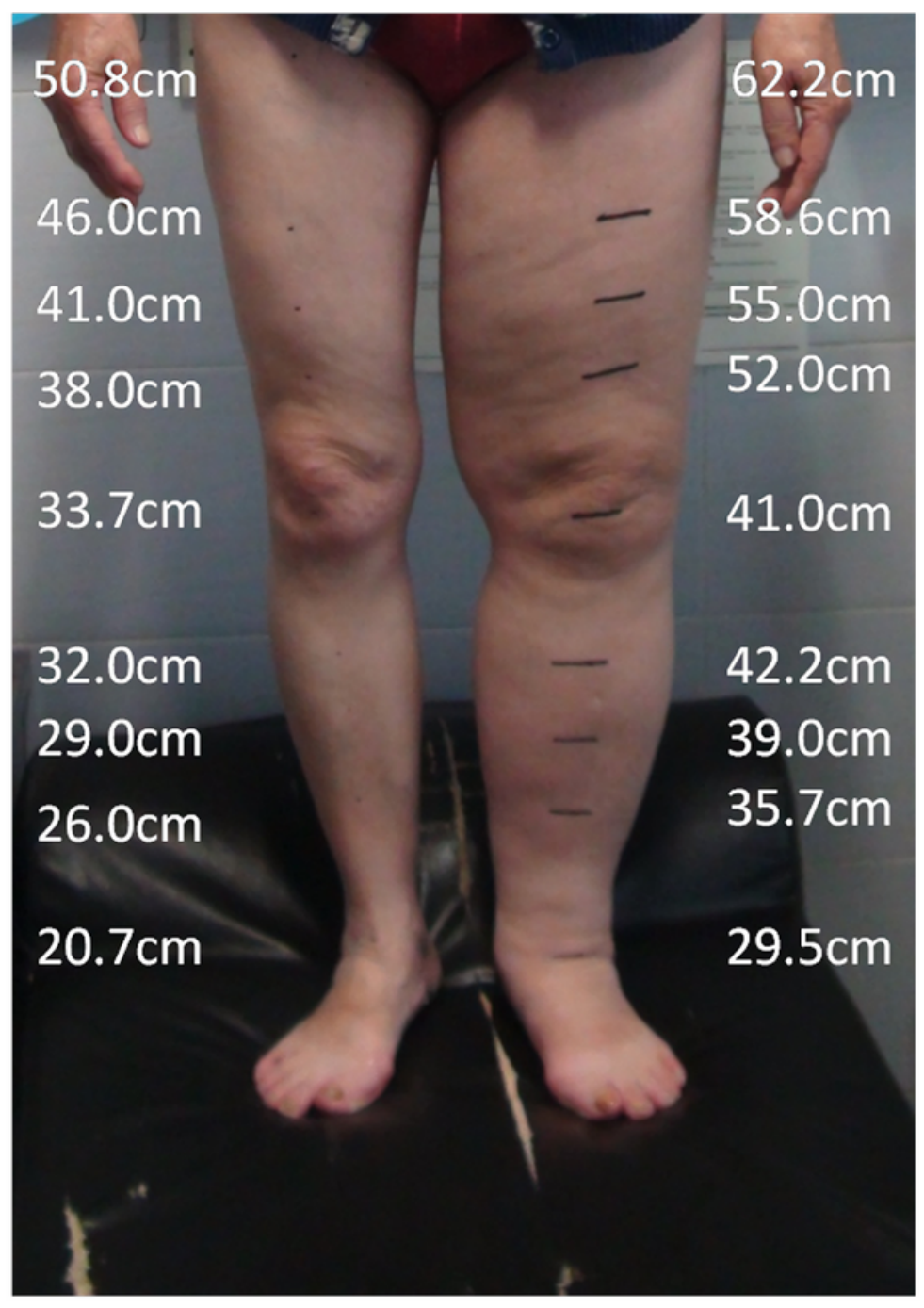

a.

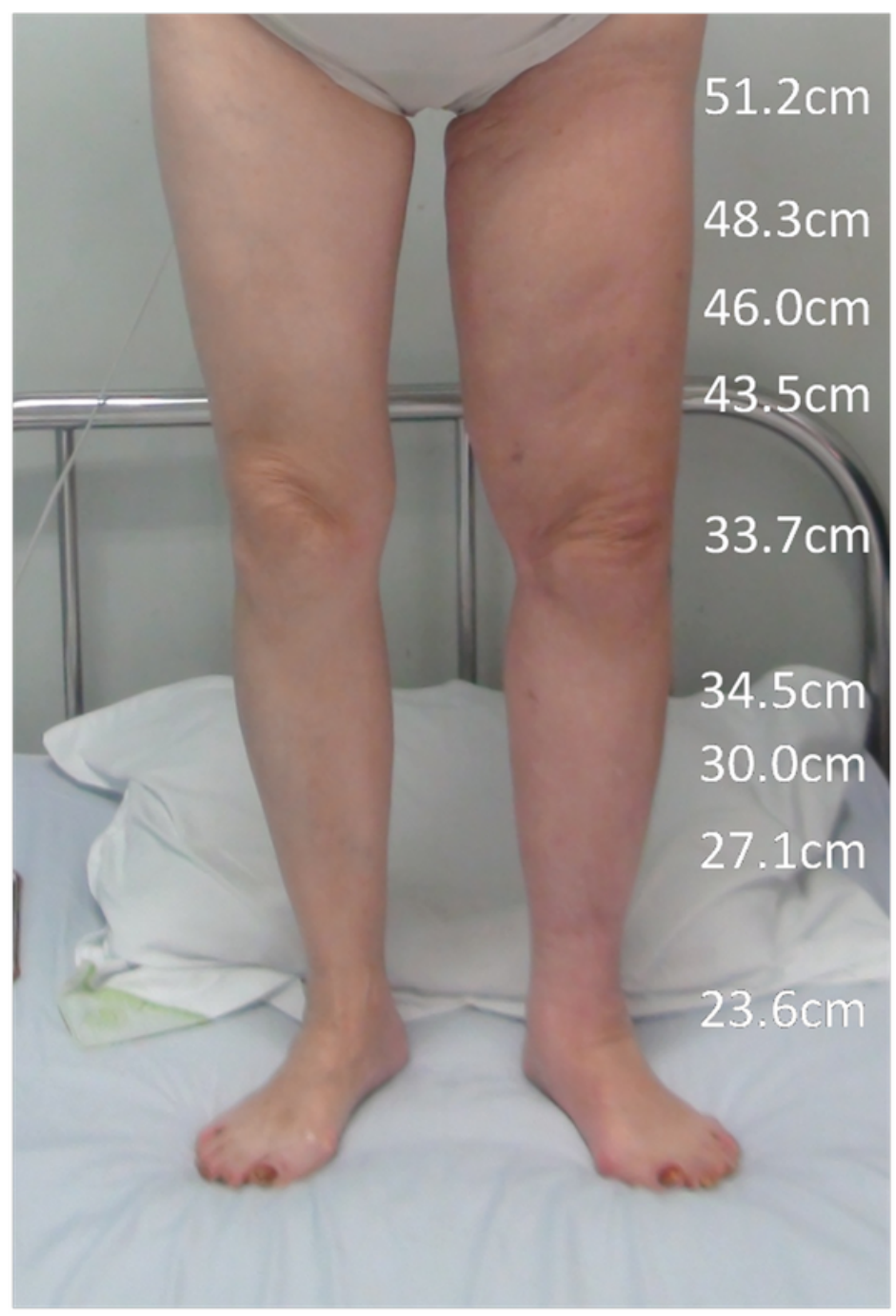

b.

Figure 1

The patient, female, 67 years old, has secondary lymphedema of the left lower extremity.A:Preoperative.B:3 months after the operation. 


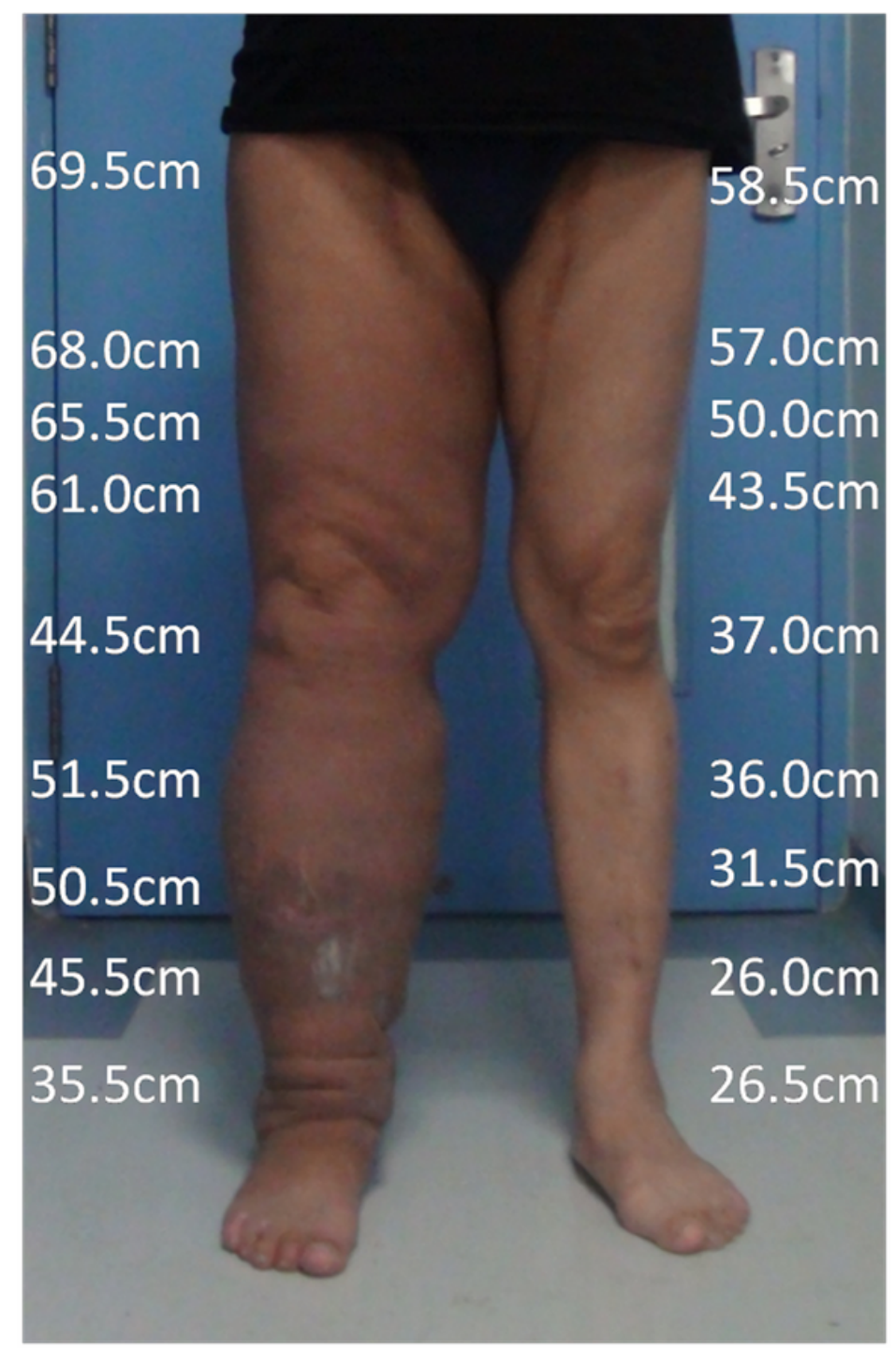

a.

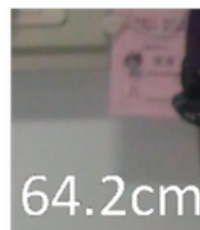

$61.3 \mathrm{~cm}$

$55.0 \mathrm{~cm}$

$48.0 \mathrm{~cm}$

$43.0 \mathrm{~cm}$

$44.5 \mathrm{~cm}$

$43.0 \mathrm{~cm}$

$40.5 \mathrm{~cm}$

$32.5 \mathrm{~cm}$

b.

\section{Figure 2}

The patient, male, 55 years old, has secondary lymphedema of the right lowerextremity.A:Preoperative.B:3 months after the operation. 